\title{
Tablas de mortalidad fetal según edad gestacional y peso del producto de la concepción
}

\section{Fetal mortality tables by gestational age and the weight of the conception outcome}

\author{
Luis Armando Martínez Barreiro'; Lorenzo Herrera León ${ }^{\text {II }}$ \\ IEspecialista de II Grado en Bioestadística. Profesor Auxiliar. Brigada Médica \\ Cubana en Honduras. Honduras. \\ IIDoctor en Ciencias Económicas. Profesor Titular. Centro de Estudios Demográficos, \\ Universidad de la Habana. La Habana, Cuba.
}

\section{RESUMEN}

Objetivos Se pretende construir tablas de mortalidad fetal con una nueva perspectiva: el uso del peso al momento de la expulsión del producto de la concepción como variable explicativa en lugar del tiempo de gestación.

Métodos El universo de estudio se conformó con todos los embarazos expulsados durante el período 1998-2002, con 22 o más semanas de gestación, en Cuba. La información utilizada se obtuvo de las bases de datos de defunciones perinatales y nacidos vivos de la Dirección Nacional de Registros Médicos y Estadísticas de Salud del Ministerio de Salud Pública de la República de Cuba y de la Oficina Nacional de Estadísticas, respectivamente. Por tratarse de una situación en la cual la temporalidad que exige la tabla de mortalidad no es unívoca en el sentido de la correspondencia peso-edad gestacional, se establecieron dos supuestos básicos: continuidad de la variable peso, lo cual no deja dudas, y condición estrictamente creciente, elemento que de asumirse en condiciones fisiológicas y clínicas favorables durante el embarazo, es aceptable.

Resultados Los resultados de esta aplicación formal de la técnica para el diseño de la tabla de mortalidad, abre una nueva perspectiva para la estimación del riesgo de muerte fetal en función del peso y en general, de variables para las cuales pueda asumirse sin demasiados riesgos, que el desarrollo fetal, bajo condiciones 
normales, sigue una trayectoria creciente.

Conclusiones Se constatan algunas equivalencias entre la sobrevivencia fetal según la edad gestacional y según el peso al momento de la expulsión del feto, hecho que pudiera constituir una nueva perspectiva en la construcción y uso de tablas de mortalidad.

Palabras clave: Tabla de mortalidad, peso al momento de la expulsión, permanencia o sobrevivencia fetal.

\begin{abstract}
Objectives It was intended to devise fetal mortality tables with a new perspective: the use of weight at the moment of expulsing the conception outcome as an explanatory variable instead of the length of gestation.

Methods The universe of study was made up of all the twenty two or over week pregnancies expulsed during the 1998-2002 period in Cuba. The information came from the perinatal death and livebirth databases of the National Division of Medical Register and Health Statistics of the Ministry of Public Health of Cuba, and of the National Office of Statistics, respectively. Since temporality demanded by the mortality table is not unique in terms of weight-gestational age correspondence; two basic assumptions were set: continuity of the weight variable that eliminates any doubt, and strictly growing condition, an element that is acceptable if assumed under favourable physiological and clinical conditions during pregnancy.

Results The results of this formal application of the technique for the design of the mortality table open up a new perspective to estimate fetal death risks as a function of weight, and generally speaking, as a function of variables for which one may assume that fetal development under normal circumstances follows an upward trajectory.

Conclusions It is observes that there are some similarities between fetal survival according to gestational age and the fetal survival according to the weight at the moment of delivering the fetus. This fact may become a new prospect for the development and use of the mortality tables.
\end{abstract}

Key words: Mortality table, weight at the moment of delivery, fetal permanence or survival.

\title{
INTRODUCCIÓN
}

La técnica de la tabla de mortalidad o de vida, se aplica esencialmente a eventos que dependen del tiempo. El ejemplo más clásico es el de la mortalidad: una persona nace y vive cierto tiempo y después muere. El tiempo de vida no es igual para todos los individuos por lo que tiene cierto carácter aleatorio.

Esta técnica, surgida primordialmente para el estudio de la mortalidad, fue usada para estudiar otros eventos como la morbilidad y la fecundidad. En estudios clínicos para medir sobrevivencia a ciertos tratamientos de enfermedades como el cáncer. 
En general fue aplicada a estudios en los que el evento de interés es de tipo no repetible, es decir, que acontece solamente una vez al individuo. ${ }^{1}$

En este quehacer, también se han diseñado tablas de mortalidad para examinar la mortalidad intrauterina en función de la edad gestacional y más recientemente en Cuba, se han hecho indagaciones sobre la sobrevivencia del feto en el útero en relación con el tiempo de gestación. ${ }^{2,3}$

En el presente trabajo se pretende construir tablas de mortalidad fetal (a partir de la vigésima segunda semana de gestación) pero con una nueva perspectiva: el uso del peso del producto de la concepción al momento de la expulsión como variable explicativa; en lugar del tiempo de gestación.

Esta tentativa permitirá realizar estimaciones de las probabilidades de muerte, según el peso del producto de la concepción, al igual que de otras funciones importantes de la tabla de mortalidad como la sobrevivencia o permanencia, y realizar comparaciones con las estimaciones obtenidas de tablas de mortalidad que usan la edad gestacional.

\section{MÉTODOS}

En el presente trabajo se han construido cohortes de embarazos a partir de la vigésima segunda semana de gestación (comienzo del período fetal) en función del peso al momento de la expulsión del producto de la concepción.

El supuesto básico subyacente, tomado como premisa fundamental se relaciona con el hecho de que desde la formación embrionaria hasta la salida del producto de la concepción, el peso es una magnitud continua y creciente, es decir, el producto de la concepción transita desde un peso aproximado de $2 \mathrm{~g}$ en la creación embrionaria) hasta el valor con que sale del vientre materno. Se descarta la posibilidad de su disminución siempre y cuando estén dadas las condiciones óptimas desde el punto de vista clínico y fisiológico durante el embarazo. ${ }^{4-6}$

Lo ideal en el embarazo es aumentar entre 9 y 15 kilos de peso en los nueve meses de gestación cuando se comienza con un peso normal. Si se comienza con un peso inferior al ideal, lo más probable es que se aumente unos kilos extras, y si se comienza con exceso de peso sería óptimo no aumentar más de 6 a 9 kilos. El aumento de peso no es parejo durante los nueve meses, por lo general en el primer trimestre no se aumenta o incluso puede bajarse de peso debido al malestar habitual de este período. Es durante el segundo trimestre y aún más, durante el último trimestre cuando hay un aumento de peso considerable, que es cuando el bebé realmente crece. ${ }^{7}$

Existen fetos con peso superiores al esperado para su edad gestacional y en otros ocurre lo contrario, su peso es deficitario para un tiempo de gestación elevado. Esto implica que la relación peso-edad gestacional no es perfectamente lineal sino que presenta cierta dispersión alrededor de valores que podrían denominarse esperados..$^{8-10}$

Para la construcción de la cohorte de embarazos según el peso se procedió de manera recurrente acorde a la metodología aplicada por Leridon, y posteriormente adaptada por Herrera León para el caso de registros vitales, pero ahora considerando el peso en vez de la edad gestacional. ${ }^{2,3}$ 
A continuación se exponen los detalles del proceso de construcción de la cohorte de embarazo:

- Los embarazos a inicio del intervalo de peso al momento de la expulsión $(p, p+1)$, $E_{p}$ se obtienen a partir de la relación recurrente:

$E_{p}=E_{p+1}+D_{p}+B_{p(1)}$

donde $D_{p}$ y $B_{p}$ denotan las salidas de fetos muertos y nacidos vivos con pesos entre $p$ y $p+1 g$ y por $E_{p+1}$, los embarazos al inicio del intervalo $(p+1, p+2)$.

-A los intervalos de la forma $(p, p+1)$ se le ha atribuido una amplitud de $500 \mathrm{~g}$.

-La relación anterior (1), se utiliza en todos los intervalos de peso, incluido el que contiene los pesos más bajos, es decir, $(0,500)$.

- Se supone que un feto expulsado con determinado peso, por ejemplo $3 \mathrm{000g}$, ha tenido necesariamente que transitar por todos los valores de peso menores a esa cifra y que además, puede haber estancamiento en la ganancia de peso (el feto puede permanecer algún tiempo sin experimentar incrementos).

- La tabla de mortalidad construida supone que el único riesgo al que está sometido cualquier embarazo es la expulsión de un feto como nacido muerto, por lo que aquellos que nacieron vivos, ahora entran en riesgo de muerte fetal (riesgo en competencia).

- El riesgo de mortalidad fetal en el intervalo $(p, p+1)$ se obtiene mediante la expresión:

$\gamma_{p}=D_{p} /\left(E_{p}-0,5 D_{p}\right)$

- El universo de estudio corresponde a las defunciones del período fetal (a partir de la vigésima segunda semana de gestación) y los nacidos vivos ocurridos en toda la nación en el período 1998-2002, también a partir de la vigésima segunda semana de gestación. En total, para el período analizado, se cuenta con 722239 nacidos vivos y 10695 defunciones fetales.

- La información anterior está contenida en las bases de datos de mortalidad perinatal y nacidos vivos, procedentes de la Dirección Nacional de Estadísticas del Ministerio de Salud Pública de Cuba (DNE) y de la Oficina Nacional de Estadísticas (ONE), respectivamente.

- Las fuentes de figuras y tablas son el resultado de la elaboración propia realizada por los autores con el paquete estadístico SPSS v.13 y MS Excel.

\section{RESULTADOS Y DISCUSIÓN}

El peso al momento de la expulsión puede decirse que guarda una relación aproximadamente lineal creciente con la edad gestacional, aunque la misma presenta un grado de dispersión que en ocasiones distorsiona un poco la relación. La figura 1 muestra el diagrama de dispersión de esta relación para las defunciones fetales y los nacidos vivos. Se observa que para las primeras la relación está mejor 
delineada que para los nacidos vivos, con una pendiente positiva mayor, que se dibuja a pesar de la dispersión que presenta la nube de puntos.

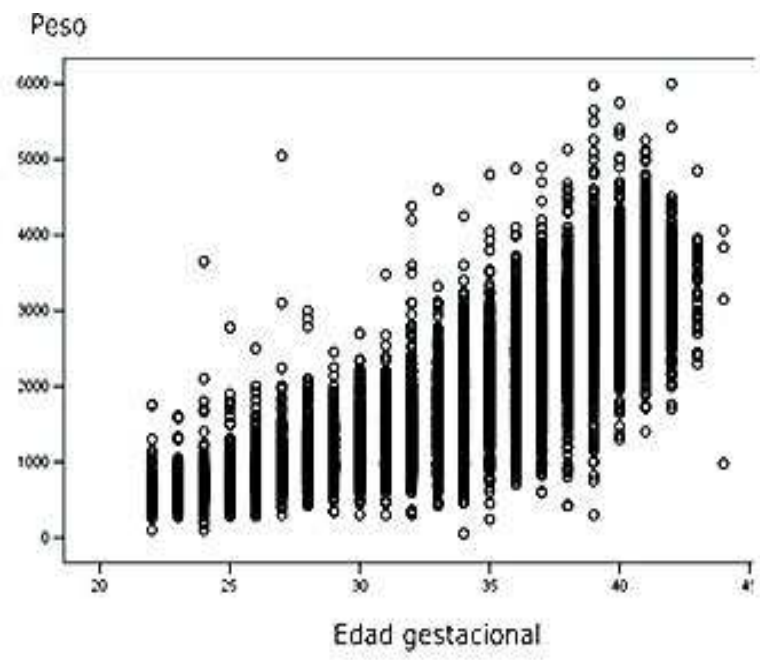

Relación peso-edad gestacional defunciones. Cuba.1998-2002.

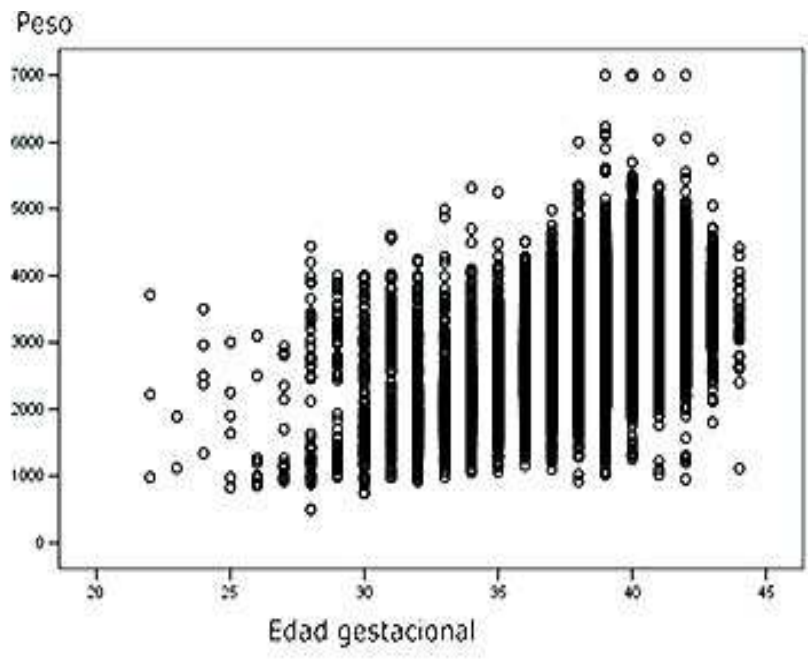

Relación peso-edad gestacional vivos. Cuba.1998-2002.

Fig. 1. Relación peso-gestacional, según resultado del embarazo. Cuba, 1998-2002.

La propia figura confirma que ha pesar de incrementarse el peso a medida que el tiempo de gestación aumenta, la dispersión se incrementa; dando lugar a la existencia de fetos con edades gestacionales bajas que presentan pesos elevados y otros con edades gestacionales elevadas, (por ejemplo, 40 o más semanas de gestación, cuyos pesos no superan los $1500 \mathrm{~g}$ ). Este hecho hace que a través de la relación descrita no sea posible determinar unívocamente el peso del feto en función del tiempo de embarazo.

Como consecuencia de lo comentado, las tablas de mortalidad construidas utilizando como variable explicativa el peso, deben interpretarse con la debida precaución, pues persiste la tendencia de vincular el peso y la edad gestacional en su interpretación.

Las tablas 1 y $\underline{2}$ exhiben las funciones más importantes utilizadas convencionalmente y entre estas, las probabilidades condicionales de muerte en cada intervalo y la función de permanencia o sobrevivencia fetal, que describen la medida en que la cohorte va menguando sus efectivos a través del tiempo de gestación o a través del tránsito por el peso. 
Tabla 1. Tabla de vida de embarazos según edad gestacional. Cuba, 1998-2002

\begin{tabular}{|c|c|c|c|c|c|c|c|}
\hline $\begin{array}{l}\text { Duración } \\
\text { (Semanas } \\
\text { completas } \\
\left(x_{1} x+1\right)\end{array}$ & $\begin{array}{c}\text { Embarazos } \\
\text { al inicio } \\
\text { del } \\
\text { intervalo }\end{array}$ & $\begin{array}{l}\text { Número } \\
\text { de } \\
\text { salidas } \\
\text { como } \\
\text { nacidos } \\
\text { vivos }\end{array}$ & $\begin{array}{c}\text { Embarazos } \\
\text { expuestos } \\
\text { al riesgo } \\
\text { de } \\
\text { mortalidad }\end{array}$ & $\begin{array}{l}\text { Número } \\
\text { de } \\
\text { embarazos } \\
\text { que } \\
\text { terminan } \\
\text { en } \\
\text { defunción }\end{array}$ & $\begin{array}{l}\text { Probabilidad } \\
\text { de muerte } \\
\text { fetal en el } \\
\text { intervalo }\end{array}$ & $\begin{array}{l}\text { Probabilidad } \\
\text { de } \\
\text { sobrevivir el } \\
\text { intervalo }\end{array}$ & $\begin{array}{l}\text { Función de } \\
\text { permanencia }\end{array}$ \\
\hline 22 & 730307 & 31 & 730291,5 & 158 & 0,0000216 & 0,999784 & 0,999784 \\
\hline 23 & 730118 & 24 & 730106 & 279 & 0,000382 & 0,999618 & 0,999402 \\
\hline 24 & 729815 & 40 & 729795 & 433 & 0,000593 & 0,999407 & 0,998809 \\
\hline 25 & 729342 & 49 & 729317,5 & 523 & 0,000717 & 0,999283 & 0,998092 \\
\hline 26 & 728770 & 87 & 728726,5 & 776 & 0,001065 & 0,998935 & 0,997030 \\
\hline 27 & 727907 & 194 & 727810 & 777 & 0,001068 & 0,998932 & 0,995965 \\
\hline 28 & 726936 & 454 & 726709 & 603 & 0,000830 & 0,999170 & 0,995139 \\
\hline 29 & 725879 & 680 & 725539 & 504 & 0,000695 & 0,999305 & 0,994447 \\
\hline 30 & 724695 & 1339 & 724025,5 & 469 & 0,000648 & 0,999352 & 0,993803 \\
\hline 31 & 722887 & 1997 & 721888,5 & 436 & 0,000604 & 0,999396 & 0,993203 \\
\hline 32 & 720454 & 2687 & 719110,5 & 488 & 0,000679 & 0,999321 & 0,992529 \\
\hline 33 & 717279 & 2789 & 7158845 & 414 & 0,000578 & 0,999422 & 0,991955 \\
\hline 34 & 714076 & 5395 & 711378,5 & 489 & 0,000687 & 0,999313 & 0,991273 \\
\hline 35 & 708192 & 7705 & 704339,5 & 403 & 0,000572 & 0,999428 & 0,990706 \\
\hline 36 & 700084 & 15559 & 692304,5 & 480 & 0,000693 & 0,999307 & 0,990019 \\
\hline 37 & 684045 & 37195 & 665447,5 & 518 & 0,000778 & 0,999222 & 0,989248 \\
\hline 38 & 646332 & 84605 & 604029,5 & 599 & 0,000992 & 0,999008 & 0,988267 \\
\hline 39 & 561128 & 172701 & 474777,5 & 646 & 0,001361 & 0,998639 & 0,986923 \\
\hline 40 & 387781 & 197791 & 288885,5 & 761 & 0,002634 & 0,997366 & 0,984323 \\
\hline 41 & 189229 & 124762 & 126848 & 603 & 0,004754 & 0,995246 & 0,979644 \\
\hline 42 & 63864 & 58114 & 34807 & 293 & 0,008418 & 0,991582 & 0,971397 \\
\hline 43 & 5457 & 5123 & 2895,5 & 30 & 0,010361 & 0,989639 & 0,961333 \\
\hline
\end{tabular}

Mediana 44

Tabla 2. Tabla de mortalidad fetal según peso al momento de la expulsión. Cuba, 1998-2002

\begin{tabular}{|c|c|c|c|c|c|c|c|}
\hline $\begin{array}{l}\text { Intervalo } \\
\text { de peso } \\
\left(P_{1} P+1\right)\end{array}$ & $\begin{array}{c}\text { Embarazos } \\
\text { al inicio } \\
\text { del } \\
\text { interualo }\end{array}$ & $\begin{array}{l}\text { Número } \\
\text { de } \\
\text { salidas } \\
\text { como } \\
\text { nacidos } \\
\text { vivos }\end{array}$ & $\begin{array}{c}\text { Embarazos } \\
\text { expuestos } \\
\text { al riesgo } \\
\text { de } \\
\text { mortalidad }\end{array}$ & $\begin{array}{l}\text { Número } \\
\text { de } \\
\text { embarazos } \\
\text { que } \\
\text { terminan } \\
\text { en } \\
\text { defunción }\end{array}$ & $\begin{array}{l}\text { Probabilidad } \\
\text { de muerte } \\
\text { fetal en el } \\
\text { interualo }\end{array}$ & $\begin{array}{l}\text { Probabilidad } \\
\text { de } \\
\text { sobrevivir el } \\
\text { intervalo }\end{array}$ & $\begin{array}{l}\text { Función de } \\
\text { permanencia }\end{array}$ \\
\hline 0 & 731441 & 1 & 731440,5 & 202 & 0,000276 & 0,999724 & 0,999724 \\
\hline 500 & 731238 & 243 & 731116,5 & 3698 & 0,005058 & 0,994942 & 0,994667 \\
\hline 1000 & 727297 & 2917 & 725838,5 & 1513 & 0,002084 & 0,997916 & 0,992594 \\
\hline 1500 & 722867 & 8292 & 718721 & 1192 & 0,001659 & 0,998341 & 0,990948 \\
\hline 2000 & 713383 & 34913 & 695926,5 & 1106 & 0,001589 & 0,998411 & 0,989373 \\
\hline 2500 & 677364 & 153145 & 600791,5 & 1187 & 0,001976 & 0,998024 & 0,987418 \\
\hline 3000 & 523032 & 298870 & 373597 & 1039 & 0,002781 & 0,997219 & 0,984672 \\
\hline 3500 & 223123 & 177461 & 134392,5 & 535 & 0,003981 & 0,996019 & 0,980752 \\
\hline 4000 & 45127 & 39234 & 25510 & 164 & 0,006429 & 0,993571 & 0,974447 \\
\hline 4500 & 5729 & 4924 & 3267 & 35 & 0,010713 & 0,989287 & 0,964008 \\
\hline 5000 & 770 & 497 & 521,5 & 15 & 0,028763 & 0,971237 & 0,936280 \\
\hline 5500 & 258 & 85 & 215,5 & 4 & 0,018561 & 0,981439 & 0,918901 \\
\hline
\end{tabular}

Alternativamente, se muestra en las figuras 2 y $\underline{3}$ estas funciones, que por añadidura presentan algunas similitudes. Los riesgos de expulsión de muerte fetal

http://scielo.sld.cu 
presentan un máximo relativo entre las duraciones 26-27 semanas de gestación, luego descienden ligeramente para hacerse crecientes, de forma aproximadamente exponencial a partir de las 37 semanas de gestación. Este comportamiento ha sido descrito por varios autores, coincidiendo con lo planteado aquí. Por su parte, las probabilidades según peso son en cierto sentido un eco de lo que sucede con la edad gestacional: un máximo relativo alrededor de los $500 \mathrm{~g}$, un descenso y luego, desde los $3000 \mathrm{~g}$ en adelante, la curva supera sus valores anteriores con un crecimiento exponencial [Yudkin PL. Risk of unexplained stillbirth at different gestational ages. Lancet. 1(8543):1192].
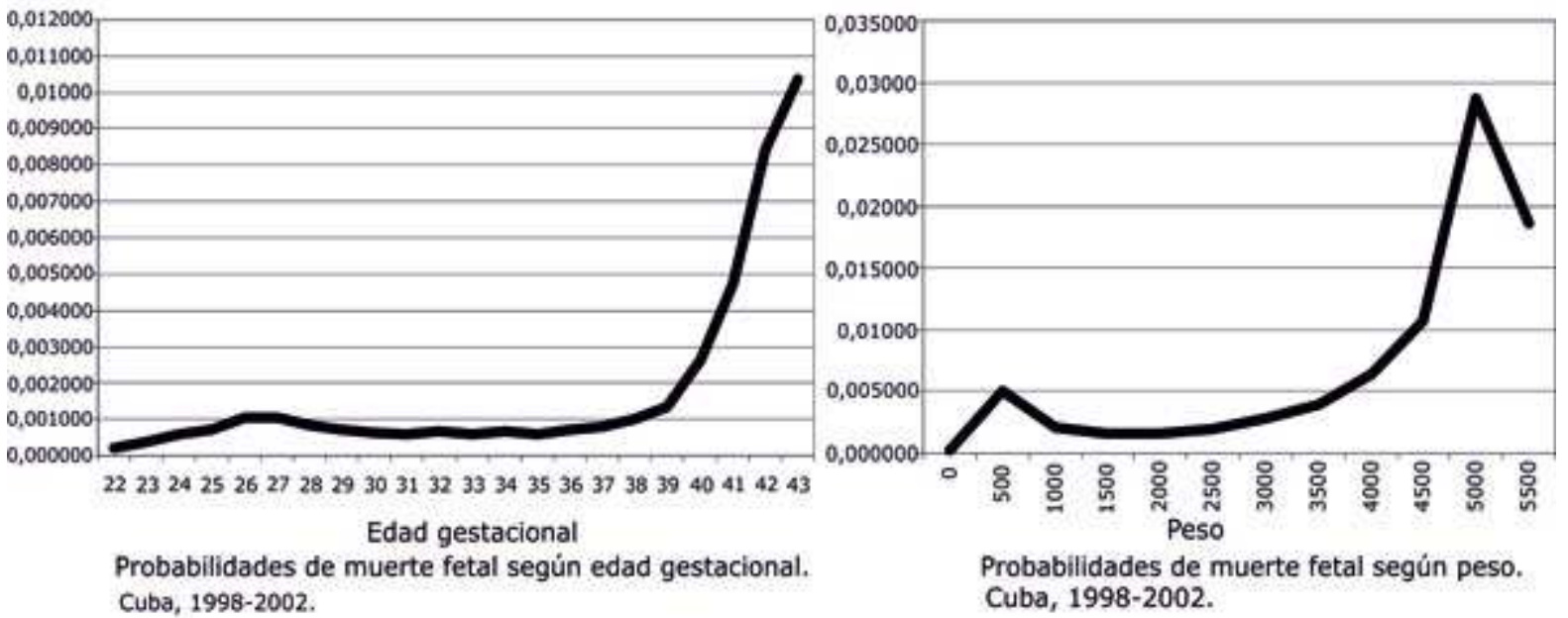

Probabilidades de muerte fetal según peso. Cuba, 1998-2002.

Fig. 2. Probabilidades de muerte según edad gestacional y peso. Cuba, 1998-2002.
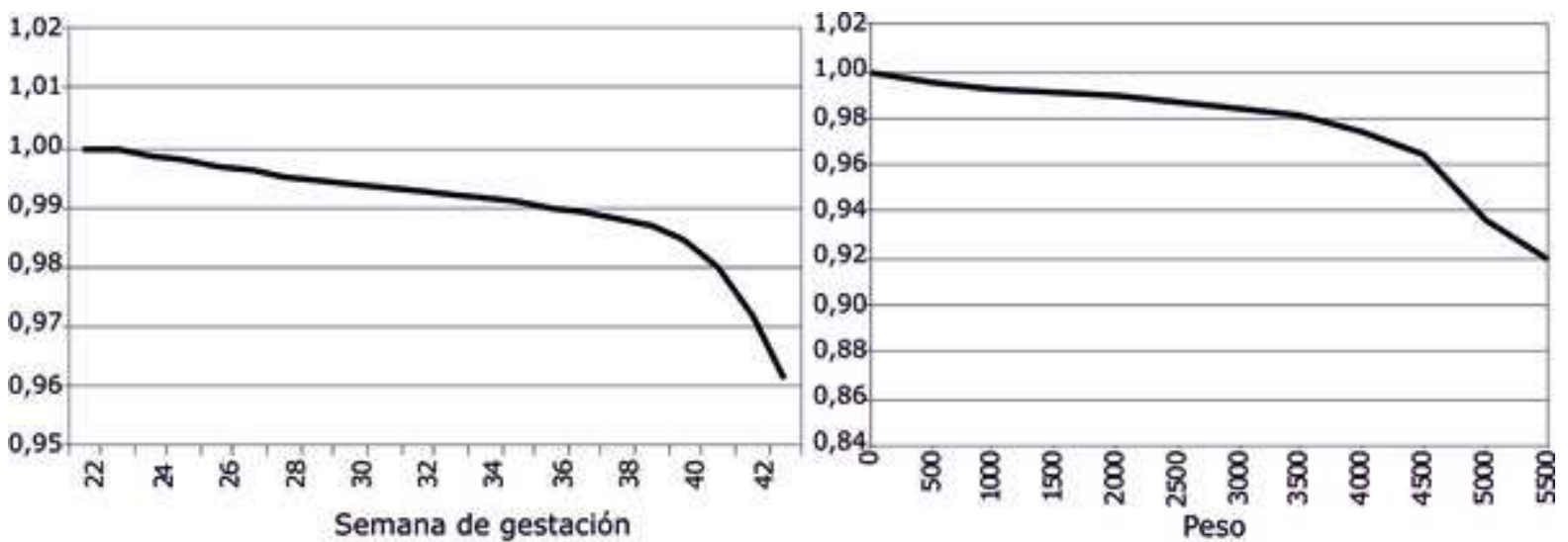

Fig. 3. Función de sobrevivencia fetal según edad gestacional y peso. Cuba, 1998-2002.

Las curvas de sobrevivencia fetal, merecen algunos comentarios previos antes de realizar un análisis más profundo. En primer lugar, un valor cualquiera de esta función representa la proporción de embarazos que aún continúan en curso (es decir, no han sido expulsados del vientre materno) para una edad gestacional dada. Es en el caso de la parte izquierda de la figura 3, para un peso dado, en el caso de la parte derecha de la propia figura. Para la gráfica de la edad gestacional, las expulsiones siguen el orden cronológico señalado por el eje horizontal, a la vez que el peso de los fetos expulsados puede variar según se mostró en la parte izquierda de la figura 1. El orden reflejado en el eje horizontal del peso, por el contrario, no implica necesariamente un orden cronológico en las salidas de los fetos, ya que, retomando un comentario precedente, existen embarazos en los cuales el producto de la concepción es de peso alto para la edad gestacional y viceversa. Es 
importante resaltar que ese eje del peso, implica una obligatoriedad en cuanto al crecimiento fetal en el sentido de que si un feto tiene determinado peso, necesariamente tuvo que transitar por todos los anteriores a ese valor.

Hay que añadir, la existencia de cierta equivalencia entre las dos curvas de permanencia fetal. A modo de ejemplo, puede observarse que para la semana 37, en la curva de permanencia según tiempo gestacional, aún queda el $99 \%$ de la cantidad original de embarazos en curso, valor de sobrevivencia que es alcanzado cuando los embarazos llegan a los

$2000 \mathrm{~g}$ de peso. También para una duración del embarazo de 41 semanas, en la cual todavía se conserva el $98 \%$ de los efectivos de la cohorte, se tiene el mismo valor para un peso de $3500 \mathrm{~g}$.

La observación de una u otra curva, denota que, muchas de las expulsiones de fetos muertos están ocurriendo en edades gestacionales y pesos propicios para el nacido vivo. Aún más, la merma de la cohorte de embarazos en ambos casos, es más acelerada en edades gestacionales y pesos posteriores a las 37 semanas (duración para la cual se supone un feto a término) y a $2000 \mathrm{~g}$, respectivamente.

\section{CONSIDERACIONES FINALES}

La construcción de tablas de mortalidad con una variable que no es estrictamente temporal como el peso al momento de la expulsión del producto de la concepción, entraña algunas particularidades. En primer lugar, debe valorarse el supuesto básico sobre el cual reposa su diseño: el peso del feto en útero es siempre una magnitud continua y creciente. La continuidad es dada como un hecho irrefutable, mientras que el carácter creciente de esa magnitud, dependerá de las condiciones en las que transcurre el embarazo. No obstante, en los autores consultados al respecto, no queda rectificada con claridad esta cuestión.

En segundo lugar, por lo general es difícil desligar la relación temporal, en el sentido que se tiende a pensar que las expulsiones de fetos para un determinado valor de peso se corresponden unívocamente con una duración de la gestación dada. Si la relación peso-edad gestacional fuera perfectamente lineal, no habría lugar a dudas al asumir tal modo de encarar el asunto, pero recuérdese que existe una dispersión del peso alrededor de un valor que podría denominarse esperado o promedio, la cual presenta una expansión al incrementarse la duración del embarazo.

Del mismo modo, ocurre con las expulsiones del producto de la concepción según las semanas de la gestación. Si bien todas están obligadas a seguir un curso cronológico estricto, no todas presentan el mismo valor del peso al momento de la expulsión o parto.

La utilidad de esta propuesta metodológica radica en la posibilidad de ser un instrumento alternativo para realizar estimaciones del riesgo de mortalidad fetal en función del peso, de manera continua. Hasta el presente, el modo más común de llevarlas a cabo consiste en el cálculo de tasas y probabilidades por categorías, que en ocasiones se apoyan en el uso de modelos multivariados de regresión. El uso reiterado acompañado de una crítica oportuna decidirá su pertinencia.

\section{REFERENCIAS BIBLIOGRÁFICAS}

http://scielo.sld.cu 
1. Ortega A. Tablas de Mortalidad. Serie E, No. 1004. San José, Costa Rica: Centro Latinoamericano de Demografía (CELADE); 1987.

2. Leridon H. Aspectos biométricos de la fecundidad humana. Serie D. No. 1031. San José, Costa Rica: Centro Latinoamericano de Demografía (CELADE); 1977.

3. Herrera L. Patrón y nivel de la sobrevivencia fetal. Cuba, 1998-2002 [tesis]. Centro de Estudios Demográficos: Universidad de La Habana; 2005.

4. Rigol O. Obstetricia y Ginecología. La Habana: Editorial Ciencias Médicas; 2005.

5. Sadler, Langman T. Embriología médica con orientación clínica. 8va ed. La Habana: Editorial Ciencias médicas; 2005.

6. ¿Cuál es el peso que debería tener el final del embarazo? [sitio en Internet]. [citado 21 Ene 2008]. Disponible en:

http://salud.infoplena.com/embarazo/aumento-normal-de-peso -durante-elembarazo

7. Embarazo semana a semana, lo que hay que saber: guía para las madres [sitio en Internet]. [citado 21 Ene 2008]. Disponible en:

http://www.abcpedia.com/embarazo/embarazo-semana-a-semana.htm

8. Solís P, Pullum SG, Frisbie WP. Demographic models of birth outcomes and infant mortality: an alternative measurement approach. Demography. 2000;37:489-98.

9. Lagos R. Índice neonatal en recién nacidos adecuado a la edad gestacional. XXVII Congreso chileno en Obstetricia y Ginecología [sitio en Internet]. [citado 21 Ene 2008]. Disponible en:

http://www.med.ufro.cl/obgin/Fronteras/vol1num1/articulos/indicepon.pdf.1997

10. Grandi C. Relación entre la antropometría materna y la ganancia de peso gestacional con el peso de nacimiento. Riesgo de peso bajo al nacer pequeño para la edad gestacional y prematurez en una población de Buenos Aires. Archivos Latinoamericanos de Nutrición [serie en Internet];53(4). [citado 21 Ene 2008]. Disponible en: http://www.scielo.org.ve/scielo.php?pid=S0004$\underline{06222003000400006 \& \text { script }=\text { sci arttext }}$

Recibido: 7 de febrero de 2009.

Aprobado: 19 de enero de 2010.

Luis Armando Martínez Barreiro. Director Docente de la Brigada Médica Cubana en Honduras. Honduras.

E-mail: lambarreiro@yahoo.cu, lorenzo@cedem.uh.cu 